\title{
Real-World Experience With Automated Insulin Pump Technology in Veterans With Type 1 Diabetes
}

\author{
Morolake Amole, MD; Loren Whyte, MS, RD, CDE; Hans K. Ghayee, DO; Fernando Bril, MD; Kenneth Cusi, MD; and
} Julio Leey-Casella, MD, MSc

Background: Advancements in diabetes technology now allow insulin pump and continuous glucose monitor (CGM) technology to be a part of usual US Department Veterans Affairs (VA) clinical care. The automated insulin pump (AIP) delivers insulin automatically based on CGM readings. In randomized clinical trials the closed-loop system has shown to improve glycemic control in children and younger adults with type 1 diabetes mellitus (T1DM) while preventing hypoglycemia. However, its safety and efficacy is less well known in older veterans with T1DM. In this VA pilot study, we aimed to assess AIP technology in the real world of an older population of veterans with T1DM followed in the outpatient setting.

Methods: Thirty-seven patients with T1DM new to AIP seen at the Malcom Randall VA Medical Center in Gainesville, Florida, were evaluated between March and December of 2018 on an Medtronic Minimed 670G Insulin Pump System. We collected demographic as well as clinical data be- fore and after the initiation of AIP, including standard insulin pump/CGM information (sensor wear, time in target glucose range, time in automated mode, other).

Results: At the time of the initiation of AIP, the mean (SD) age of patients was 59.1 (14.4) years; 35 identified as male and 2 as female. The mean (SD) duration of T1DM was 25.3 (12.0) years. Patients transitioned from either insulin injections or other non-AIP pump to AIP safely - there was no increase in hypoglycemia, and the mean (SD) hemoglobin $A_{1 c}$ decreased from $7.6 \%(0.8)$ to $7.3 \%(0.80)$ by the second follow-up visit.

Conclusion: In this real-world study, AIP use was both safe and viable as a tool for T1DM management with older veterans. This technology further engaged veterans in monitoring their blood sugars and achieving more optimal glycemic control. Future long-term, larger studies are much needed in this setting.
Author affiliations can be found at the end of the article.

Correspondence: Julio Leey-Casella (julio.leey-casella@va.gov)

Fed Pract. 2021;38(7). Published online July 22. doi:10.12788/fp.0156 nsulin pump technology has been available since the 1970s. Innovation in insulin pumps has had significant impact on the management of diabetes mellitus (DM). In recent years, automated insulin pump technology (AIP) has proven to be a safe and effective way to treat DM. It has been studied mostly in highly organized randomized controlled trials (RCTs) in younger populations with type 1 DM (T1DM). ${ }^{1-3}$

One of the challenges in DM care has always been the wide variations in daily plasma glucose concentration that often cause major swings of hyperglycemia and hypoglycemia. Extreme variations in blood glucose have also been linked to adverse outcomes, including poor micro- and macrovascular outcomes. ${ }^{4,5}$ AIP technology is a hybrid closed-loop system that attempts to solve this problem by adjusting insulin delivery in response to real-time glucose information from a continuous glucose monitor (CGM). Glucose measurements are sent to the insulin pump in real time, which uses a specialized algorithm to determine whether insulin delivery should be uptitrated, down-titrated, or suspended. ${ }^{6}$

Several studies have shown that AIP technology reduces glucose variability and increases the percentage of time within the optimal glucose range. ${ }^{1-3,7}$ Its safety is especially indicated for pa- tients with long-standing DM who often have hypoglycemia unawareness and recurrent episodes of hypoglycemia. ${ }^{7}$ Safety is the major advantage of the hybrid closed-loop system as long duration of DM makes patients particularly prone to emergency department (ED) visits and hospitalizations for severe hypoglycemia. ${ }^{8}$ Recurrent hypoglycemia also is associated with increased cardiovascular mortality in epidemiologic studies. ${ }^{9}$

Safety was the primary endpoint in the pivotal trial in a multicenter clinical study where 124 participants (mean age, 37.8 years; DM duration, 21.7 years; hemoglobin $\mathrm{A}_{1 \mathrm{c}}\left[\mathrm{HbA}_{1 \mathrm{c}}\right], 7.4 \%$ ) were monitored for 3 months while using a hybrid closed-loop pump, similar to the one used in our study..$^{10}$ Remarkably, there were no device-related episodes of severe hypoglycemia or ketoacidosis. There was even a small but significant difference in $\mathrm{HbA}_{1 \mathrm{c}}$ (7.4\% at baseline, $6.9 \%$ at 3 months) and of the time in target range measured by CGM from $66.7 \%$ at baseline to $72.2 \%$ at 3 months). However, the mean age of the population studied was young (mean age, 37.8 years). It is unclear how these results would translate for a population of older patients with T1DM. Moreover, use of AIP systems have not been systematically tested outside of carefully controlled studies, as it would be in middle-aged veterans followed in 
outpatient US Department of Veterans Affairs (VA) clinics. Such an approach in the context of optimal glucose monitoring combined with use of structured DM education can significantly reduce impaired awareness of hypoglycemia in patients with T1DM of long duration. ${ }^{11}$

This is the first study to assess the feasibility of AIP technology in a real-world population of older veterans with T1DM in terms of safety and acceptability, because AIP has just recently become available for patient care in the Veterans Health Administration (VHA). This group of patients is of particular interest because they have been largely overlooked in earlier studies. They represent an older population with long-standing DM where hypoglycemia unawareness is often recurrent and incapacitating. In addition, long-standing DM makes optimal glycemic control mandatory to prevent microvascular complications.

\section{METHODS}

In this retrospective review study,, we examined available data in patients with T1DM at the Malcom Randall VA Medical Center diabetes clinic in Gainesville, Florida, between March and December of 2018 who agreed to use AIP. In this clinic, the AIP system was offered to T1DM patients when the 4-year warranty of a previous insulin pump expired, they had frequent hypoglycemic events, or they were on multiple daily injections and were proficient with carbohydrate counting and adjusting insulin doses and willing to use an insulin pump. Veterans were trained on AIP use by a certified diabetes educator and pump trainer in sessions that lasted 2 to 4 hours depending on previous experience with AIP. Institutional review board approval was obtained at the University of Florida.

Demographic and clinical data before and after the initiation of AIP were collected, including standard insulin pump/CGM information for the Medtronic 670G and Guardian 3 Sensor AIPs. Several variables were evaluated, including age, gender, year of DM diagnosis, time of initiation of AIP, $\mathrm{HbA}_{10}$, download data (percentage sensor wear, time in automated mode and manual mode, time in/above/below range, bolus information, insulin use, average sensor blood glucose, average meter blood glucose, pump settings), weight, body mass index (BMI), glucose meter information, history of hypoglycemia unawareness.

The primary outcome for this study was safety as assessed by percentage of time below target range on glucose sensor (time below target range
TABLE 1 Baseline Demographics $(\mathrm{N}=37)$

\begin{tabular}{lc} 
Characteristics & Results \\
\hline Age, mean (SD), y & $59.1(14.4)$ \\
\hline $\begin{array}{l}\text { Gender, No. } \\
\text { Male } \\
\text { Female }\end{array}$ & 35 \\
\hline Duration of diabetes mellitus, mean (SD), y & $25.3(12.0)$ \\
\hline Weight, kg & $83.3(14.3)$ \\
\hline Body mass index, mean (SD) & $26.1(4.2)$ \\
\hline Hemoglobin $\mathrm{A}_{1 \mathrm{c}}, \%$ & $7.6(0.8)$
\end{tabular}

is defined as $<70 \mathrm{mg} / \mathrm{dL}$ ). We also addressed the secondary endpoint of efficacy as the percentage of time in-range defined as blood glucose per glucose sensor of $70 \mathrm{mg} / \mathrm{dL}$ to $180 \mathrm{mg} / \mathrm{dL}$ (efficacy), percentage of glucose sensor wear, and $\mathrm{HbA}_{1 c}$.

\section{Statistics}

Comparisons of changes in continuous variables between groups were performed by an analysis of covariance (ANCOVA), adjusting for baseline levels. Fisher exact test $\left(\chi^{2}\right)$ and unpaired $t$ test were used to compare group differences at baseline for categorical and continuous variables, respectively, while Wilcoxon rank sum test was used for nonnormally distributed values. Changes in continuous measures within the same group were tested by paired $t$ test or Wilcoxon matched-pairs signed rank test when applicable. Analyses were performed using Stata 11.0.

\section{RESULTS}

Thirty-seven veterans with T1DM using AIPs in 2018 were evaluated at baseline and at follow up visits (Tables 1 and 2). Time frame for follow-up was approximately 3 months, although there was some variation. Of note, the mean weight and $\mathrm{BMI}$ corresponded to mostly lean individuals, consistent with the diagnosis of T1DM.

Time below target range hypoglycemia (sensor glucose $<70 \mathrm{mg} / \mathrm{dL}$ ) remained low at each follow-up visit (both 1.5\%). Percentage of time in automated mode increased from first to second follow-up visit after initiation of AIP ( $41 \%$ vs $53 \%, P=.06$ ). Percentage of sensor wear numerically increased from first to second follow-up visit ( $75 \%$ vs $85 \%, P=.39$ ), same as time in range, defined as sensor glucose 70 to $180 \mathrm{mg} / \mathrm{dL}$, from first to second follow-up visit ( $70 \%$ vs $73 \%$, 
$P=$.09). Time above range, defined as sensor glucose $>180 \mathrm{mg} / \mathrm{dL}$, demonstrated a strong trend toward decreasing between follow-up appointments $(29 \%$ to $25 \% ; P=.09)$. $\mathrm{HbA}_{1 \mathrm{c}}$ decreased from $7.6 \%$ to $7.3 \%(P=.005)$.

About half of the patients (18 of 37) reported hypoglycemia unawareness before the initiation of the 670G AIP. On follow-up visit 61\% (11 of 18) reported significant improvement in awareness. Of the remaining 18 patients who reported normal awareness before automated mode, $17 \%$ (3 of 18) described a new onset unawareness.

\section{DISCUSSION}

This study evaluated the safety of adopting a new DM technology in the real world of an outpatient VA clinic. To the best of our knowledge, this is the first study evaluating the use of AIP specifically in a population of middle-aged veterans with longstanding T1DM. After a mean 7 months of followup, participants accepted AIP use as evidenced by increased sensor wear over time and experienced improvements in DM measures that indicate successful use (ie, time in automated mode, which represents reduced glycemic variability). These results show success of an AIP approach in a demographically older group of patients.

AIP has been shown to have positive effects on glycemic control such as time in target glucose range (goal $\geq 70 \%$ ). In our relatively small pilot study, there was trend for an improvement in the time in range from the first to second clinical follow-up visit, suggesting true patient involvement with the use of the device. Studies involving overall younger cohorts have proved that AIP technology is safe and efficacious for outpa-

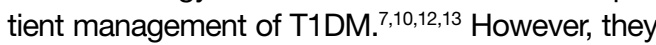
were all conducted under the safety of a research setting, and trials enrolled a younger population believed to adapt with more ease to this new technology. Tauschmann and colleagues performed a multicenter, parallel randomized controlled trial that compared hybrid closed-loop AIP therapy with sensor-augmented pump therapy in patients with suboptimal T1DM control. ${ }^{12} \mathrm{Re}$ sults showed that the hybrid closed-loop system increased the time that the glucose concentration was within the target range $(70-180 \mathrm{mg} / \mathrm{dL})$ from $54 \%$ in the sensor-augmented pump group to $65 \%$ on the closed-loop system $(P<.001)$. A small but significant improvement in $\mathrm{HBA}_{1 \mathrm{c}}$ (from $8.0-7.4 \%)$ and low rates of hypoglycemia (2.6\% of time below $70 \mathrm{mg} / \mathrm{dL}$ ) were also noted. ${ }^{12}$

A similar benefit was observed in a 2019 landmark study by Brown and colleagues of 168 patients with T1DM at 7 university medical centers who were treated for 6 months with either a closed-loop system (closed-loop group) or a sensor-augmented pump (control group) in a parallel-group, unblinded, randomized trial study. ${ }^{13}$ Mean (SD) time in the target range increased in the closed-loop group from $61 \%$ (17) at baseline to $71 \%$ (12) during the 6 months. $\mathrm{HbA}_{1 \mathrm{c}}$ decreased from 7.4 to $7.1 \%$ and time $\leq 70 \mathrm{mg} / \mathrm{dL}$ was just $1.6 \%$. However, only $13 \%$ of patients were aged $\geq 40$ years in the study by Tauschmann and colleagues, and mean age was 33 years in the Brown and colleagues study. ${ }^{12,13}$ In contrast, the mean (SD) age in our study was 59 (14) years. Our pilot study also showed comparable, or somewhat better results, as mean time in target range was $72 \%, \mathrm{HbA}_{1 \mathrm{c}}$ was $7.3 \%$, and time $\leq 70 \mathrm{mg} / \mathrm{dL}$ was just $1.5 \%$.

In the only other single-center study in adults with T1DM (mean age 45 years), Faulds and colleagues evaluated changes in glycemic control and adherence in patient using the same hybrid closed-loop system. ${ }^{14}$ Treatment resulted in a decrease in $\mathrm{HbA}_{1 \mathrm{c}}$ compared with baseline similar to our study, most notably for patients who had higher baseline $\mathrm{HbA}_{1 \mathrm{c}}$. However, over its short duration (6 to 12 weeks), there was decreased time in automated mode in study patients, likely due to treatment burden. Our study in older patients showed a similar reduction in $\mathrm{HbA}_{1 \mathrm{c}}$ from baseline up to the 7-month visit but with increased sensor wear and time in automated mode.

There are many possible reasons for improved time in target range in our older population. Contrary to common belief that older age may be a barrier to adopting complex technology, it is likely that older age and longer duration of DM motivates adherence to a therapy that reduces glucose swings, offers a greater sense of safety and control, and improves quality of life. This is underscored by improvements over time in sensor wear and time in automated mode, measures of adherence, and successful AIP management. In support of a motivation factor to adopt insulin pump therapy in patients with long-standing T1DM, Faulds and colleagues found that older age and higher baseline $\mathrm{HbA}_{1 \mathrm{c}}$ were associated with less time spent in hypoglycemia. ${ }^{14}$

The close supervision of patients by a certified diabetes educator and pump trainer may have helped improve glycemic control. Veterans received initial training, weekly follow-ups 
TABLE 2 Glycemic Control Results at Follow-Up Visits

\begin{tabular}{lccc} 
Measures & First Follow-up & Second Follow-up & $P$ value \\
\hline Time on insulin pump, mean (SD), d & $70.2(78.2)$ & $133.1(123.0)$ & .001 \\
\hline Time wearing sensor, mean (SD), \% & $75.3(28.2)$ & $85.1(18.4)$ & .39 \\
\hline $\begin{array}{l}\text { Time in mode, mean (SD), \% } \\
\quad \begin{array}{l}\text { Manual } \\
\text { Automated }\end{array}\end{array}$ & $59.1(40.0)$ & $46.4(42.3)$ & .06 \\
\hline $\begin{array}{l}\text { Time in range, mean (SD), \% } \\
\leq 70 \text { mg/dL }\end{array}$ & $41.5(40.4)$ & $53.2(42.1)$ & .06 \\
\hline $\begin{array}{l}70-180 \mathrm{mg} / \mathrm{dL} \\
>180 \mathrm{mg} / \mathrm{dL}\end{array}$ & $1.5(1.8)$ & $1.5(1.9)$ & .09 \\
\hline Sensor plasma glucose, mean (SD), mg/dL & $29.1(13.4)$ & $73.2(12.1)$ & .99 \\
\hline Meter plasma glucose, mean (SD), mg/dL & $157.0(17.1)$ & $153.1(17.1)$ & .09 \\
\hline Hemglobin $\mathrm{A}_{1 \mathrm{c}}$, mean (SD), \% & $181.2(34.5)$ & $181.1(29.2)$ & .90 \\
\hline
\end{tabular}

for 4 to 5 visits, and then bimonthly visits. There was also good access to the DM care team through a secure VA messaging system. This allowed for prompt troubleshooting and gave veterans the support they needed for the successful technology adoption.

The use of real-time CGM led to improvements in hypoglycemia unawareness. The nature of automated insulin delivery not only allows the patient to use a immediate CGM, but automatically lowers the delivery of insulin, further minimizing the risk of hypoglycemia. ${ }^{15}$ This combined approach explains the improvement in selfreported hypoglycemia unawareness in our cohort which decreased by $61 \%$. As in our study, very recently Pratley and colleagues reported in a 6-month follow-up study that the greatest benefit of CGM was not the $-0.3 \%$ improvement of glycemic control (similar in magnitude to our study) but the $47 \%$ decrease in the primary outcome of CGM-measured time in hypoglycemia. ${ }^{16}$

Hybrid closed-loop insulin delivery improves glucose control while reducing the risk of hypoglycemia. There is consensus that this approach is cost-effective and saves resources in the management of these complex patients, so prone to severe microvascular complications and hypoglycemia. ${ }^{17,18} \mathrm{~A}$ recent analysis by Pease and colleagues concluded that the hybrid closed-loop system was safer and more costeffective when compared with the current standard of care, comprising insulin injections and capillary glucose testing. ${ }^{19}$ This held true even after several sensitivity analyses were performed, including baseline glycemic control, treatment effects, technology costs, age, and time horizon. This is relevant to the VHA, which at all times must consider the most cost-effective approach. Therefore, while there is no such debate about the cost-effectiveness of AIP technology for younger adults with T1DM, this study closes the knowledge gap for middle-aged veterans. ${ }^{7,10,12,13}$ The current study demonstrates that even for older patients with long-standing T1DM, when proper access to supplies and support services are made available, treatment is associated with considerable success.

Finally, AIP is well suited for telehealth applications. Data can be uploaded remotely and sent to VA health care providers, which can facilitate care without the need to travel. Distance is often a barrier for access and optimal care of veterans. The current COVID-19 pandemic is another barrier to access that may persist in the near future and adds value to AIP management.

There were a few challenges with use of AIP. Although transition to AIP was smooth for most patients already on insulin pump therapy, several noted requests for calibration in the middle of the night in automated mode, which affected sleep. Also, AIP technology requires some computer literacy to navigate the menu and address sensor calibrations, which can be a challenge for some. Based on our results, we would recommend AIP in veterans who are appropriately trained in carbohydrate counting, understand the principles of insulin therapy, and are able to navigate a computer screen menu. Most T1DM patients already 
using insulin pump meet those recommendations, thus, they are good candidates.

\section{Limitations}

There are some limitations to our study. The small sample size and single-center nature prevent generalization. Also, the veteran population cannot be extrapolated to other populations. For instance, the majority of the patients in this study were male.

\section{CONCLUSIONS}

We report that an AIP approach for patients with long-standing T1DM is well accepted and engages patients into monitoring their blood sugars and achieving better glycemic control. This was achieved with minimal hypoglycemia in a population where often hypoglycemia unawareness makes DM care a challenge. Future studies within the VHA are needed to fully assess the long-term benefits and cost-effectiveness of this technology in veterans.

\section{Author affiliations}

Morolake Amole is an Endocrinology Fellow; Hans Ghayee is an Associate Professor of Medicine; Fernando Bril is a Internal Medicine resident; Kenneth Cusi is the Chief of the Division of Endocrinology, Diabetes and Metabolism; and Julio Leey-Casella is an Assistant Professor of Medicine; all at the University of Florida in Gainesville. Loren Whyte is a Certified Diabetes Educator and pump trainer; Kenneth Cusi is Endocrine Faculty; Hans Ghayee is Section Chief of Endocrinology; and Julio Leey-Casella is an Endocrinologist; all at Malcom Randall VA Medical Center.

\section{Author disclosures}

The authors report no actual or potential conflicts of interest with regard to this article.

\section{Disclaimer}

The opinions expressed herein are those of the authors and do not necessarily reflect those of Federal Practitioner, Frontline Medical Communications Inc., the US Government, or any of its agencies. This article may discuss unlabeled or investigational use of certain drugs. Please review the complete prescribing information for specific drugs or drug combinations -including indications, contraindications, warnings, and adverse effects-before administering pharmacologic therapy to patients.

\section{References}

1. Saunders A, Messer LH, Forlenza GP. MiniMed 670 G hybrid closed loop artificial pancreas system for the treatment of type 1 diabetes mellitus: overview of its safety and efficacy. Expert Rev Med Devices. 2019;16(10):845-853. doi:10.1080/17434440.2019.1670639

2. Beato-Víbora PI, Quirós-López C, Lázaro-Martín L, et al. Impact of sensor-augmented pump therapy with predictive low-glucose suspend function on glycemic contro and patient satisfaction in adults and children with type 1 diabetes. Diabetes Technol Ther. 2018;20(11):738-743. doi:10.1089/dia.2018.0199

3. De Ridder F, den Brinker M, De Block C. The road from intermittently scanned continuous glucose monitoring to hybrid closed-loop systems. Part B: results from ran- domized controlled trials. Ther Adv Endocrinol Metab. 2019;10:2042018819871903. Published 2019 Aug 30. doi: $10.1177 / 2042018819871903$

4. Monnier L, Colette C, Wojtusciszyn A, et al. Toward defining the threshold between low and high glucose variability in dabetes. Diabetes Care. 2017;40(7):832-838. doi:10.2337/dc16-1769

5. Monnier L, Colette C, Owens DR. The application of simple metrics in the assessment of glycaemic variability. Diabetes Metab. 2018;44(4):313-319. doi:10.1016/j.diabet.2018.02.008

6. Thabit $\mathrm{H}, \mathrm{Hovorka}$ R. Coming of age: the artificial pancreas for type 1 diabetes. Diabetologia. 2016;59(9):1795-1805. doi:10.1007/s00125-016-4022-4

7. Anderson SM, Buckingham BA, Breton MD, et al. Hybrid closed-loop control is safe and effective for people with type 1 diabetes who are at moderate to high risk for hypoglycemia. Diabetes Technol Ther. 2019;21(6):356-363. doi:10.1089/dia.2019.0018

8. Liu J, Wang R, Ganz ML, Paprocki Y, Schneider D, Weatherall J. The burden of severe hypoglycemia in type 1 diabetes. Curr Med Res Opin. 2018;34(1):171-177. doi:10.1080/03007995.2017.1391079

9. Rawshani A, Sattar N, Franzén S, et al. Excess mortality and cardiovascular disease in young adults with type 1 diabetes in relation to age at onset: a nationwide, registerbased cohort study. Lancet. 2018;392(10146):477-486. doi:10.1016/S0140-6736(18)31506-X

10. Bergenstal RM, Garg S, Weinzimer SA, et al. Safety of a hybrid closed-loop insulin delivery system in patients with type 1 diabetes. JAMA. 2016;316(13):1407-1408. doi:10.1001/jama.2016.11708

11. Little SA, Speight J, Leelarathna L, et al. Sustained reduction in severe hypoglycemia in adults with type 1 diabetes complicated by impaired awareness of hypoglycemia: two-year follow-up in the HypoCOMPaSS randomized clinical trial. Diabetes Care. 2018;41(8):16001607. doi:10.2337/dc17-2682

12. Tauschmann $M$, Thabit $H$, Bally $L$, et al. Closedloop insulin delivery in suboptimally controlled type 1 diabetes: a multicentre, 12-week randomised trial [published correction appears in Lancet. 2018 Oct 13;392(10155):1310]. Lancet. 2018;392(10155):1321-1329. doi:10.1016/S0140-6736(18)31947-0

13. Brown SA, Kovatchev BP, Raghinaru D, et al. Six-month randomized, multicenter trial of closed-loop control in type 1 diabetes. N Engl J Med. 2019;381(18):1707-1717. doi:10.1056/NEJMoa1907863

14. Faulds ER, Zappe J, Dungan KM. Real-world implications of hybrid close loop (HCL) insulin delivery system. Endocr Pract. 2019;25(5):477-484. doi:10.4158/EP-2018-0515

15. Rickels MR, Peleckis AJ, Dalton-Bakes C, et al. Continuous glucose monitoring for hypoglycemia avoidance and glucose counterregulation in long-standing type 1 diabetes. J Clin Endocrinol Metab. 2018;103(1):105-114. doi:10.1210/jc.2017-01516

16. Pratley RE, Kanapka LG, Rickels MR, et al. Effect of continuous glucose monitoring on hypoglycemia in older adults with type 1 diabetes: a randomized clinical trial. JAMA. 2020;323(23):2397-2406. doi:10.1001/jama.2020.6928

17. Bekiari E, Kitsios K, Thabit H, et al. Artificial pancreas treatment for outpatients with type 1 diabetes: systematic review and meta-analysis. BMJ. 2018;361:k1310. Published 2018 Apr 18. doi:10.1136/bmj.k1310

18. American Diabetes Association. Addendum. 7. Diabetes technology: standards of medical care in diabetes-2020. Diabetes Care. 2020;43(suppl 1):S77-S88. Diabetes Care. 2020;43(8):1981. doi:10.2337/dc20-ad08c

19. Pease A, Zomer E, Liew D, et al. Cost-effectiveness analysis of a hybrid closed-loop system versus multiple daily injections and capillary glucose testing for adults with type 1 dabetes. Diabetes Technol Ther. 2020;22(11):812-821. doi:10.1089/dia.2020.0064 